\section{Gastrointestinal sodium absorption, microbiome, and hypertension}

\section{Ricardo S. Mishima, Adrian D. Elliott, Prashanthan Sanders and Dominik Linz}

We read with interest the Review by Marques et al. (Beyond gut feelings: how the gut microbiota regulates blood pressure. Nat. Rev. Cardiol. http://dx.doi.org/10.1038/ nrcardio.2017.120;2017) ${ }^{1}$. In their article, the authors provide a comprehensive overview of the current understanding of the interaction between dietary intake and the composition of the gut microbiota and its role in the regulation of blood pressure. They summarize several human studies showing that the human gut microbiome in patients with hypertension harbours lower microbial diversity and an increased Firmicutes:Bacteroidetes ratio accompanied by decreases in acetateproducing and butyrate-producing bacteria ${ }^{2,3}$. Interestingly, similar changes in the microbiome are also observed in genetic animal models of hypertension ${ }^{2,4,5}$. Although these observations cannot prove causality, targeted modification of the gut microbiota might be a novel adjunct treatment strategy for hypertension. Interventions such as oral antibiotic treatment ${ }^{2,5}$ and a high-fibre $\operatorname{diet}^{6}$ have been shown to attenuate high blood pressure and rebalance the dysbiotic gut microbiome in various hypertensive rodent models.
Modulation of the gastrointestinal intraluminal milieu by selective pharmacological inhibition of intestinal absorption of dietary ions, glucose, fat, or proteins might offer an alternative approach to control the gut microbiome. The proximal colon is an important site of dietary ion absorption. Sodium uptake is critically regulated by the sodium/hydrogen exchanger 3 (NHE3). Previously, we showed that pharmacological inhibition of intestinal NHE3 using an orally nonabsorbable, specific NHE3 inhibitor increased faecal sodium excretion and water content. This change was associated with a sustained reduction in systolic blood pressure and attenuation of cardiac hypertensive end-organ damage in hypertensive rats ${ }^{6,7}$. Although we did not study the microbiome after gut-restricted NHE3 inhibition, modulation of the intraluminal intestinal milieu might be a promising approach to influence the composition of gut microorganisms. Importantly, nonabsorbable and, therefore, nonsystemic drugs have fewer off-target systemic effects and, consequently, less drugdrug interaction and toxicity, and fewer adverse effects. Further studies are needed to investigate the feasibility and efficacy of altering the gut microbiome composition by oral treatment with nonabsorbable drugs specifically targeting ion transporters such as NHE3 or sodium/glucose cotransporter 1 in the gut.

Ricardo S. Mishima, Adrian D. Elliott, Prashanthan Sanders and Dominik Linz are at the Centre for Heart Rhythm Disorders, South Australian Health and Medical Research Institute (SAHMRI), University of Adelaide and Royal Adelaide Hospital, Adelaide 5000, Australia.

Correspondence to D.L. dominik.linz@adelaide.edu.au

doi: 10.1038/nrcardio.2017.159 Published online 13 Oct 2017

1. Marques, F. Z., Mackay, C. R. \& Kaye, D. M. Beyond gut feelings: how the gut microbiota regulates blood pressure. Nat. Rev. Cardiol. http://dx.doi.org/10.1038/ nrcardio. 2017.120 (2017).

2. Yang, T. et al. Gut dysbiosis is linked to hypertension. Hypertension 65, 1331-1340 (2015).

3. Li, J. et al. Gut microbiota dysbiosis contributes to the development of hypertension. Microbiome 5 14 (2017).

4. Santisteban, M. M. et al. Hypertension-linked pathophysiological alterations in the gut. Circ. Res. 120, 312-323 (2017).

5. Marques, F. Z. et al. High fibre diet and acetate supplementation change the gut microbiota and prevent the development of hypertension and heart failure in DOCA-salt hypertensive mice. Circulation 135, 964-977 (2017).

6. Linz, D. et al. Antihypertensive and laxative effects by pharmacological inhibition of sodium proton-exchanger subtype 3-mediated sodium absorption in the gut. Hypertension 60, 1560-1567 (2012).

7. Linz, B. et al. Inhibition of NHE3-mediated sodium absorption in the gut reduced cardiac end-organ damage without deteriorating renal function in obese spontaneously hypertensive rats. J. Cardiovasc. Pharmacol. 67, 225-231 (2016).

Competing interests statement

The authors declare no competing interests. 